\title{
Assessing the representativeness of thermal comfort in outdoor spaces
}

\author{
R. Cocci Grifoni ${ }^{1}$, M. Pierantozzi ${ }^{2}$, S. Tascini ${ }^{1}$ \& G. Passerini ${ }^{2}$ \\ ${ }^{I}$ School of Architecture and Design, Camerino University, Italy \\ ${ }^{2}$ Department of Energetics, Politechnic University of Marche, Italy
}

\begin{abstract}
This paper presents preliminary findings of an outdoor thermal comfort study conducted in an urban area to evaluate the representative Predicted Mean Vote index. Thermal comfort in outdoor urban spaces is often faced with the task of using large amounts of data that yields meaningful information concerning the thermal sensation. It is essential to interpret correctly meteorological and thermal comfort data. In particular, it is important to interpret data using an appropriate statistical analysis, and the analysis of thermal comfort presupposes a synthesis of information derived from a series of temporal data. It is indispensable to deal with realistic data and an actual day should be considered, but the widely used average day is not an actual day. On the contrary, the representative day is made of the actual data of the day, in the period considered, where the sum of the mean-square differences among its monitored quantities, averaged within each hour, and the same quantities for all other days at the same hour, is minimised. The goal of this research is to assess the representativeness of the thermal comfort indices provided using a representative day technique. Specifically, a new tool has been developed using a powerful and useful environment for symbolic and numerical computing and data visualization such as Wolfram MathematicaTM, aiming at linking information computed by a bio-climate model to the representative day technique. The possibility of assessing the diurnal variation of PMV thermal comfort index by introducing the Representative Day technique has been evaluated in order to gather information on the correlation between thermal comfort and meteorological parameters. A case study has been analysed in order to improve the microclimate in an outdoor space located in a typical Mediterranean area and a comparison with CFD code, namely ENVI-MET, has been reported. This technique can prove to be a very
\end{abstract}


important tool for identifying both anomalous and standard behaviours of comfort indices within the selected period in outdoor urban spaces.

Keywords: representative day, predicted mean vote, predicted percentage of dissatisfied, outdoor thermal comfort, thermo fluid dynamic analysis.

\section{Introduction}

Thermal comfort in outdoor spaces is important for assessment studies of urban quality of life. The parameters that interfere with thermal comfort in urban spaces can generally be considered similar to indoor space parameters, but they are more extended and variable, so that the understanding of comfort conditions in outdoor spaces has been the object of many studies [1]. There is a strong public interest in creating attractive open spaces. The acceptance and use of open spaces is influenced by the microclimatic conditions offered to the people, whereas the microclimate as such, as well as the thermal sensation, heavily depend on the urban design, and show a meaningful temporal and spatial variation.

The climate of the outdoor urban space has additionally important influences on the energy consumption of the cities and the processes that create these climates are very complex. Therefore, the most precise way to calculate or assess the impact of changes is using numerical methods, although there already exist several models able to deal with the complexity of urban structures and even to take into account human thermal comfort.

A lot of thermal indices have been reported in literature; common applications are: the Predicted Mean Vote (PMV) [2], Perceived Temperature [3], Physiological Equivalent Temperature (PET) [4], and Standard Effective Temperature (SET) [5]. All these thermal indices are well documented and include important meteorological and thermo-physiological parameters $[6,7]$. The advantage of these thermal indices is that they require the same meteorological input parameters: air temperature, air humidity, wind speed, short and long wave radiation fluxes.

In this study, PMV thermal comfort index has been used for assessing its diurnal variation by introducing the representative day technique in order to obtain information on the correlation between thermal comfort and meteorological parameters.

The microclimatic analysis of an urban space must consider different conditions such as solar incidence and radiation exchanges, local characteristics of winds, topography, vegetation and the presence of water. Beyond these factors, the urban design, the morphology of the buildings, the characteristics of the surfaces and the behaviour of the individuals are also factors that influence the thermal conditions of these spaces.

As a case study, the city of Ancona was chosen because it represents an example of a typical Mediterranean city located in central Italy (Marche region), characterized by long and hot summers and mild winters.

The investigations in this study are focussed almost solely on the summer period (June to September) of a meteorological data series (2006 to 2010) 
because climatic hot-stress in the urban open spaces mainly occurs during this season of the year. On the contrary, thermal cold stress only occurs during a few winter days.

\section{Representative thermal comfort index}

\subsection{PMV thermal comfort index}

The most widely used thermal comfort index is the predicted mean vote (PMV) index developed by Fanger and Toftum [8]. This thermal comfort index is a function of two human variables and four environmental variables, i.e. clothing insulation, human activity, air temperature, air relative humidity, air velocity and mean radiant temperature. The values of the PMV index range from 3 to +3 , which corresponds to the occupant's feeling from cold to hot, while the null value of PMV means neutral.

Table 1: $\quad$ Valuation scale of the thermal environment.

\begin{tabular}{|c|c|}
\hline PMV & $\begin{array}{c}\text { Thermal Environment } \\
\text { Evaluation }\end{array}$ \\
\hline+3 & Hot \\
\hline+2 & Warm \\
\hline+1 & Slightly warm \\
\hline$-0.5<$ PMV $<+0.5$ & Comfortable \\
\hline-1 & Cool \\
\hline-2 & Cold \\
\hline-3 & Extremely cold \\
\hline
\end{tabular}

The thermal stress established by the PMV is based on the steady state of heat exchange between the body and the environment. It is an empirical equation used to evaluate the mean vote on a rating scale of thermal comfort in a large population. To develop a curve and get average results, a sufficient number of people were exposed to different environments at different spans of time. The term PPD represents the predicted percentage of people dissatisfied with each PMV. As PMV shifts from zero, in either the positive or negative direction, the value of PPD increases.

The heat balance equation is based on the conservation of energy:

$$
M+W+R+C+E_{D}+E_{R D}+E_{S W}+S=0
$$

where

$M$ : the metabolic rate (internal energy production),

$W$ : the physical work output,

$R$ : the net radiation of the body,

$C$ : the convective heat flux, 
$E_{\mathrm{D}}$ : the latent heat flux to evaporate water diffused through the skin, $E_{\mathrm{RD}}$ : the sum of heat fluxes for heating and humidifying the air breathed in, $\mathrm{E}_{\mathrm{Sw}}$ : the heat flux due to sweat evaporation, $\mathrm{S}$ : the storage heat flow for heating or cooling the body mass.

Predicted Mean Vote (PMV), as the integrated partial derivative, is the most widely used thermal comfort index today.

\subsection{Representative day}

The "representative day" [9] is a 24 hour data set, actually recorded at a field station characterised by the fewest differences with respect to all the 24 hour measurements of that station's temporal series: that is to say, the daily series whose sum of the squared differences over 24 hours turns out to be the smallest compared to all the other days of the period considered.

It is constituted by the actual data of the day, in the period considered.

The least square matrix can be written as:

$$
A_{i j}=\sum_{k=1}^{24}\left(c_{k i}-c_{k j}\right)^{2} \quad i, j=1,2 \ldots N
$$

where $N$ is the number of days in the period of time for which the representative day is calculated and $c_{k i}$ is the pollutant concentration of the $i$-th day at the $k$-th hour.

$A_{i}$ indicates the sum of all the squared residuals of the $i$-th line (or column, $A_{i j}$ being a symmetrical matrix with all zeros in the main diagonal):

$$
A_{i}=\left(\sum_{j=1}^{N} A_{i j}\right)
$$

The representative day (RD) is the one with the minimum sum, i.e. the $i$-th day where $A_{i}$ is the smallest of the quantities obtained:

$$
\min (\mathrm{Ai}) \Rightarrow \mathrm{RD}
$$

The methodology introduced allows the evaluation of the "least representative day" (LRD), that is the day which maximises the sum of squared residuals:

$$
\max \left(A_{i}\right) \Rightarrow \mathrm{LRD}
$$

Usually, the least representative day corresponds to an anomalous situation of pollutant dispersion or meteorological condition.

The "representativeness" of a representative day can be mathematically expressed by the adimensional index (DI):

$$
D I=\frac{\sqrt{\sum_{i=1}^{N} \sum_{k=1}^{24}\left(\Gamma_{k}-c_{i k}\right)^{2}}}{\sqrt{\sum_{i=1}^{N} \sum_{k=1}^{24}\left(\overline{c_{k}}-c_{i k}\right)^{2}}}
$$


where $\overline{c_{k}}$ is the mean hourly concentration of the typical day at the $k$-th hour and $\Gamma_{k}$ is the mean hourly concentration of the representative day at the $k$-th hour.

It is an adimensional quantity closer to unity for the most representative day in the period observed. When DI is equal to 1 , this value denotes that the most representative day coincides with the typical day.

In the same way the least representative day can also be normalized, but the value of DI will be greater than 1, showing the low degree of representativeness of the estimated day.

\section{3 representative Predicted Mean Vote (rPMV)}

The algorithm proposed was based on defining thermal comfort by using the PMV index. For the analysis of the PMV a new program written in Wolfram Mathematica language has been implemented. The choice was determined by the fact that this software is a general purpose language that suited our needs.

The rPMV (representative Predicted Mean Vote) algorithm developed takes as input weather data of a specific region and calculates the PMV and PPD of both the representative day and the least representative day, then compares them with the values of the typical day.

The market offers many software to calculate the comfort indices described above, the novelty of this instrument is that it does not only calculates individually all meteorological parameters for a typical day or a representative day, and the thermal comfort indices as well as PMV and PPD, but it also links them, highlighting their interdependence. Moreover, the trends of these quantities can be simulated in relation to different variables, also using interactive tools for data manipulation.

In detail, the algorithm is based on weather data for the area considered in different formats into matrix structures and analysis of weather data and evaluations of the representative day, least representative day and mean day.

Finally, the program generates both numerical and graphical comparing reports so as to carry out an accurate analysis of data, which can be exported either in numerical or graphical formats in different types of files.

To calculate the PMV and the PPD the indications provided in the technical standard ISO 7730 [10] below have been followed:

$$
\begin{aligned}
\mathrm{PMV}=[0.303 \cdot & \operatorname{Exp}(-0.036 \cdot \mathrm{M})+0.028] \\
& \cdot\left\{(\mathrm{M}-\mathrm{W})-3.05 \cdot 10^{-3}\right. \\
& \cdot\left[5733-6.99 \cdot(\mathrm{M}-\mathrm{W})-\mathrm{p}_{\mathrm{a}}\right] \\
& -0.42 \cdot[(\mathrm{M}-\mathrm{W})-58.15] \\
& -1.7 \cdot 10^{-5} \cdot \mathrm{M} \cdot\left(5867-\mathrm{p}_{\mathrm{a}}\right) \\
& -0.0014 \cdot \mathrm{M} \cdot\left(34-\mathrm{t}_{\mathrm{a}}\right)-3.96 \\
& \cdot 10^{-8} \cdot \mathrm{f}_{\mathrm{cl}} \\
& \cdot\left[\left(\mathrm{t}_{\mathrm{cl}}+273\right)^{4}-\left(\overline{\mathrm{t}}_{\mathrm{r}}+273\right)^{4}\right] \\
& \left.-\mathrm{f}_{\mathrm{cl}} \cdot \mathrm{h}_{\mathrm{c}} \cdot\left(\mathrm{t}_{\mathrm{cl}}-\mathrm{t}_{\mathrm{a}}\right)\right\}
\end{aligned}
$$


840 The Sustainable City VII, Vol. 2

with

$$
\begin{aligned}
\mathrm{t}_{\mathrm{cl}}=35.7-0.028 & \cdot(\mathrm{M}-\mathrm{W})-\mathrm{I}_{\mathrm{cl}} \\
\cdot & \cdot\left\{3.96 \cdot 10^{-8} \cdot \mathrm{f}_{\mathrm{cl}}\right. \\
\cdot & \cdot\left[\left(\mathrm{t}_{\mathrm{cl}}+273\right)^{4}-\left(\overline{\mathrm{t}}_{\mathrm{r}}+273\right)^{4}\right]+\mathrm{f}_{\mathrm{cl}} \cdot \mathrm{h}_{\mathrm{c}} \\
& \left.\cdot\left(\mathrm{t}_{\mathrm{cl}}-\mathrm{t}_{\mathrm{a}}\right)\right\}
\end{aligned}
$$

$$
\begin{gathered}
\mathrm{h}_{\mathrm{c}} \\
=\left\{\begin{array}{l}
2.38 \cdot\left|\mathrm{t}_{\mathrm{cl}}-\mathrm{t}_{\mathrm{a}}\right|^{0.25} \quad, 2.38 \cdot\left|\mathrm{t}_{\mathrm{cl}}-\mathrm{t}_{\mathrm{a}}\right|^{0.25}>12.1 \cdot \sqrt{\mathrm{v}_{\mathrm{ar}}} \\
12.1 \cdot \sqrt{\mathrm{v}_{\mathrm{ar}},} \quad 2.38 \cdot\left|\mathrm{t}_{\mathrm{cl}}-\mathrm{t}_{\mathrm{a}}\right|^{0.25}<12.1 \cdot \sqrt{\mathrm{v}_{\mathrm{ar}}}
\end{array}\right. \\
f_{c l}= \begin{cases}1.00+1.290 I_{c l} & , I_{c l} \leq 0.078 m^{2} \cdot K / W \\
1.05+0.645 I_{c l} & , I_{c l}>0.078 m^{2} \cdot K / W\end{cases}
\end{gathered}
$$

and for PPD:

$$
\begin{aligned}
\mathrm{PPD}=100-95 & \\
& \cdot \operatorname{Exp}\left(-0.03353 \cdot \mathrm{PMV}^{4}\right. \\
& \left.-0.2179 \cdot \mathrm{PMV}^{2}\right)
\end{aligned}
$$

where

$M$

Metabolic rate

$$
W / m^{2}
$$

W Effective mechanical power

$$
W / m^{2}
$$

$I_{c l}$

Clothing insulation

$$
m^{2} \cdot K / W
$$

$\overline{t_{r}} \quad$ Mean radiant temperature

${ }^{\circ} \mathrm{C}$

$t_{c l} \quad$ Clothing surface temperature

${ }^{\circ} \mathrm{C}$

$t_{a}$

Air temperature

${ }^{\circ} \mathrm{C}$

$f_{c l} \quad$ Clothing surface area factor

$v_{a r} \quad$ Relative air velocity

$$
m / s
$$

$h_{c}$

Convective heat transfer coefficient

$W /\left(m^{2} \cdot K\right)$

$p_{a} \quad$ Water vapour partial pressure

$\mathrm{Pa}$ 


\section{The case study}

The evaluations of rPMV were carried out for Ancona in the Marche region, a location in central Italy at $43^{\circ} 35^{\prime} 56^{\prime \prime} \mathrm{N}, 13^{\circ} 30^{\prime} 39^{\prime \prime} \mathrm{E}$ and $16 \mathrm{~m}$. above sea level. The climate in Marche is categorised as Mediterranean, with hot, humid summers and cool winters.

During the typical summer months (from early June and to late September) the air temperature can reach high values and the daily temperature amplitude can be relatively wide. The atmospheric moisture content sometimes reaches very high levels. On the contrary, winters are short and cold, particularly at night (reaching freezing point).

Weather data from a meteorological station belonging to ASSAM (Agenzia Servizi Settore Agroalimentare Marche, Fig. 1) in Agugliano (Ancona) have been used to gather daily and monthly mean values of summer months of the main meteorological parameters.

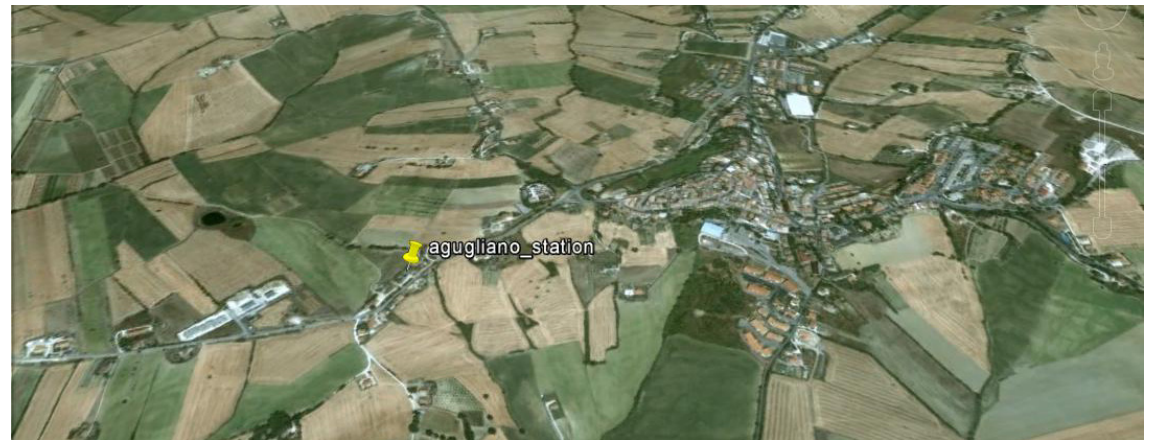

Figure 1: Weather station location.

In order to evaluate the usefulness of the Representative Day technique, summer monthly periods were analysed over four years (2006-2010).

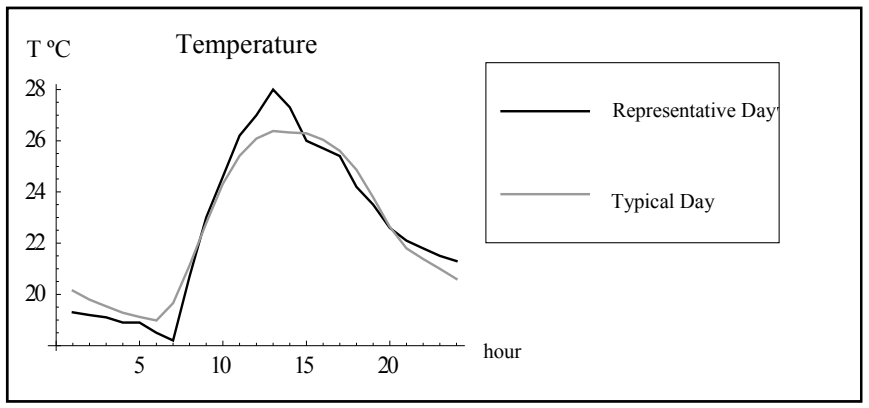

Figure 2: Representative day and typical day for temperature (15 August 2006) during summer 2006. 
The typical day, the representative day and the least representative day have been evaluated and, while both the typical and the representative day proceed in the same way (Fig. 2), the least representative day shows a different trend as for the rPMV thermal comfort index, as observed in previous studies [11].

The possibility to study a few, rather paradigmatic scenarios, suggestive of singular or standard conditions, would be of considerable help, at least in reducing the amount of data needed to shape up the study of the actual scenarios.

The calculated rPMV indices and meteorological data measured during June, July, August and September 2006 have been employed as an application example.

Figure 3 shows the data related to rPMV thermal comfort index:

The representative day for the PMV (rPMV) and the typical day (TD) present the same course, although with higher values of comfort index in the central part of the day.

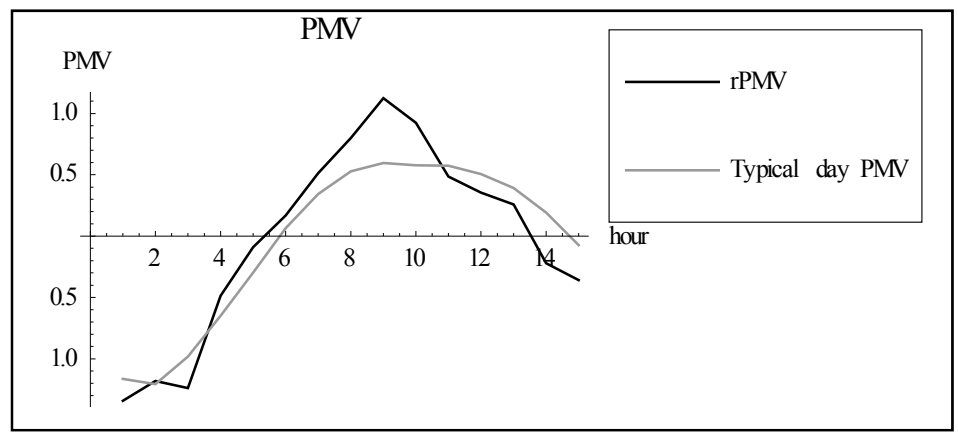

Figure 3: $\quad$ Representative day for PMV (15 August 2006) and typical day for PMV during summer 2006.

\section{Discussion}

The above results were compared with simulated results obtained with the threedimensional numerical model ENVI-met 3.0 [12], which simulated the microclimatic changes within suburban environments in a defined spatial and temporal resolution. The study area is a typical suburban zone (Agugliano, Ancona province) precisely a peripheral area where the selected weather station is located. The simulation domain includes fields with arable crops and permeable areas. The region, with built-up areas for residences, business and offices, is characterized by a relatively low density increase,

The simulation was started at 6:00 LST when most atmospheric processes are slow and a very low wind speed value was considered $(1.8 \mathrm{~m} / \mathrm{s})$ with temperature at dawn $\left(18.9^{\circ} \mathrm{C}\right)$ accordingly to meteorological data of the representative day (15 ${ }^{\text {th }}$ August, 2006).

ENVI-met is a three-dimensional non-hydrostatic microclimate model which is able to simulate climate in urban areas with a typical grid resolution of 0.5 to 
10 meters in space. It calculates the dynamics of microclimate during a diurnal cycle (up to 48 hours) using fundamental laws of fluid dynamics and thermodynamics. Main prognostic variables of the program are wind speed and direction, air temperature and humidity, turbulence, radiative fluxes, bioclimatology, and gas and particle dispersion.

The simulations were carried out during daytime, because daylight hours represent the period of day when people usually attend outdoor spaces. For the ENVI-met simulations, the study area has been transformed in a model grid with $120 \times 60 \times 20$ cells, with a $8 \mathrm{~m} \times 10 \mathrm{~m} \times 2 \mathrm{~m}$ resolution, resulting in a total area of $960 \times 600 \mathrm{~m}$ in the horizontal extension.

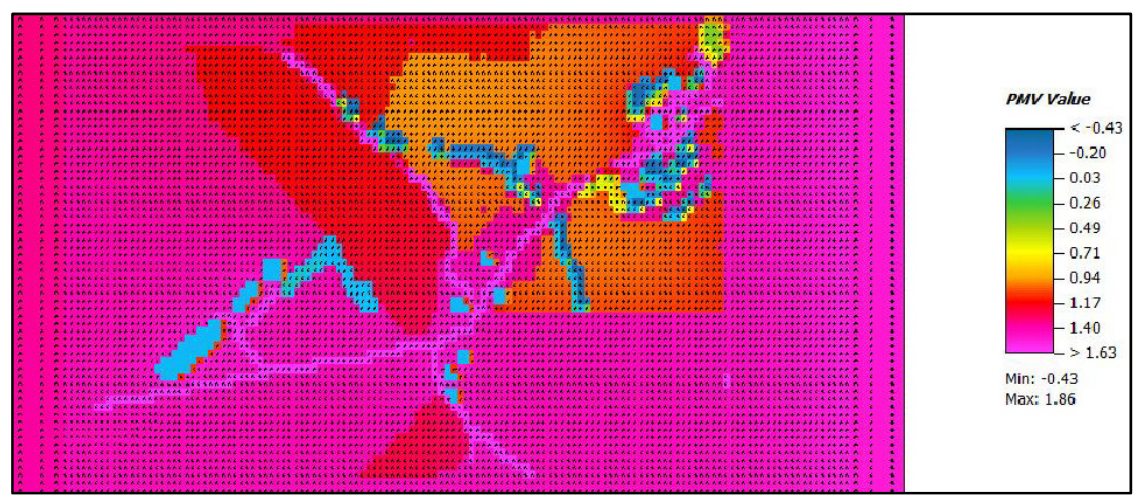

Figure 4: $\quad$ Map of PMV index (ENVI-met simulation).

Figure 4 depicts the ENVI-met output (PMV index) for the simulated summer scenario (14.00LST) and figure 5 compares the behaviour of simulated PMV with evaluated rPMV during daytime.

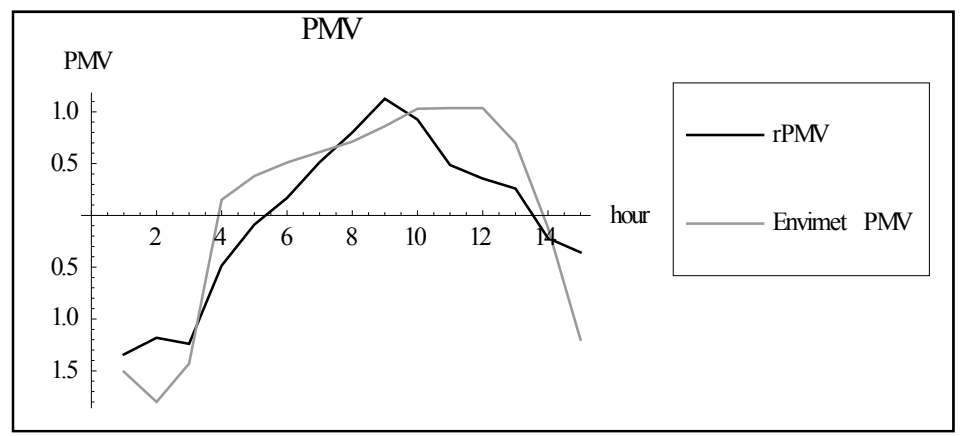

Figure 5: $\quad$ The predicted mean vote PMV simulated by ENVI-met plotted against evaluated rPMV. 
For this comparison, an adjusting factor had been set for the global radiation to fit with the measured data of global radiation provided by the urban weather station.

Considering the complexity of thermal environment, rPMV tool is found to represent efficiently the trends of thermal comfort values.

In addition to the PMV value, ENVI-met provides the associated PPD (Predicted Percentage of Dissatisfied) value which represents the percentage of people who would be dissatisfied with the given climate conditions. PPD values have a linear relationship with PMV values, in fact they can be directly transformed into each other.

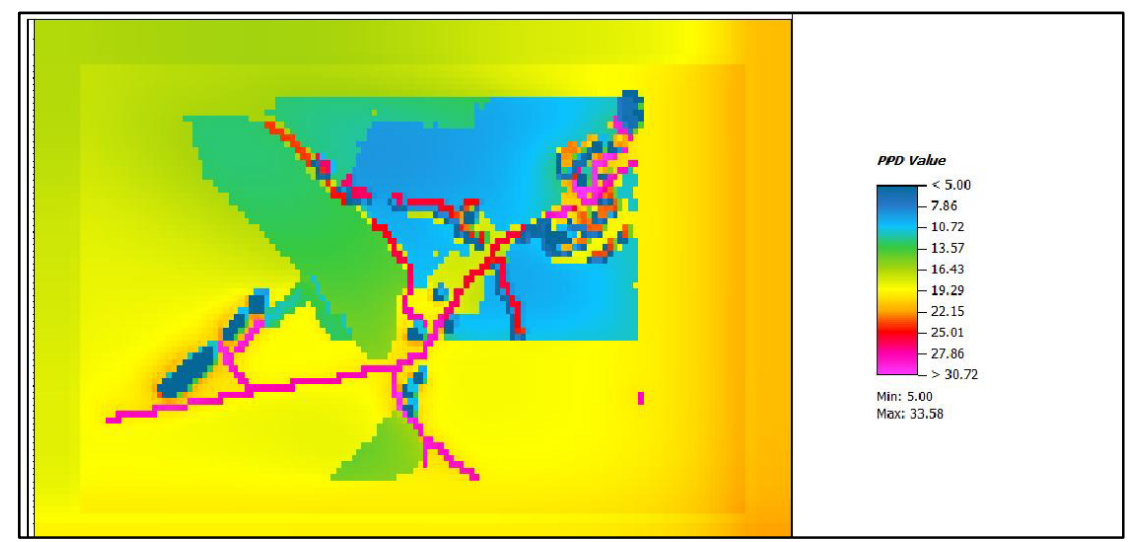

Figure 6: $\quad$ Map of PPD index (ENVI-met simulation).

Figure 6 shows that the PPD map (14 LST) has the same spatial structure as the PMV map, while figure 7 compares the behaviour of simulated PPD with evaluated rPPD during daytime.

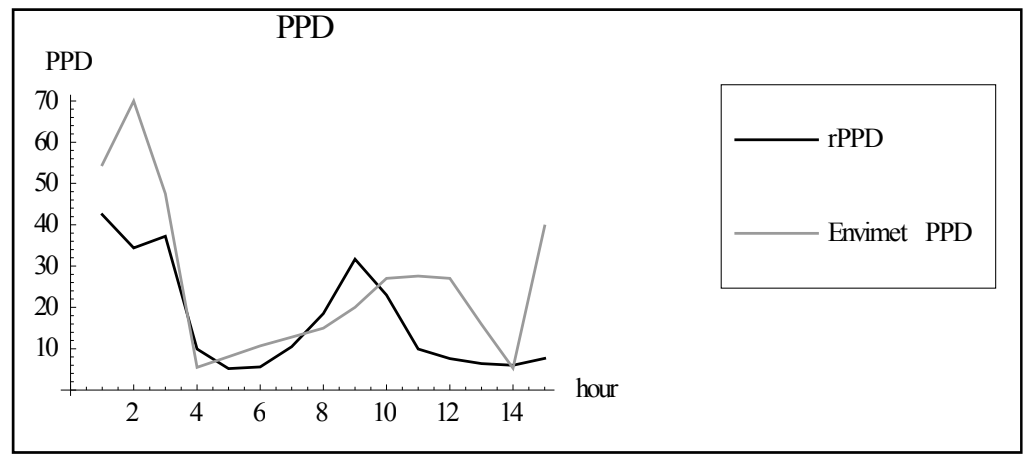

Figure 7: The Predicted Percentage Dissatisfied PPD simulated by ENVI-met plotted against evaluated rPPD. 


\section{Conclusions}

The main objective of this work was to develop and apply a user-friendly tool (rPMV) for the calculation of thermal comfort indices (PMV, PPD) in representative environmental conditions. The main advantages of the rPMV tool derive from the fact that it allows the identification of the actual day on which the representative trend occurred and, thus, information on the meteorological and thermal comfort parameters that characterised it.

The good agreement between the representative and the typical day is a further indication that the representative day should be preferred to interpret thermal comfort behaviour. The results from the Envi-met simulations, presented in this study, clearly show that a general coherence between simulated and evaluated values is evident. This observation leads to consider the proposed tool very interesting in evaluating outdoor thermal comfort indices.

\section{References}

[1] Nikolopoulou, M., Rediscovering the Urban Realm and Open Spaces (RUROS). CENTRE FOR RENEWABLE ENERGY SOUCES (CRES), Org. Greece, 2004.

[2] Fanger, P.O. Thermal comfort. McGraw-Hill, New York,1972.

[3] Tinz, B., Jendritzky, G., Europa- und Weltkarten der gefühlten Temperatur, Chmielewski, F.-M., Foken, Th. (Ed.) Beiträge zur Klima- und Meeresforschung, Berlin und Bayreuth, 2003.

[4] Matzarakis, A., Mayer, H., and Iziomon, M.G. Applications of a universal thermal index: physiological equivalent temperature, Int. J. Biometeorol. 43: 76-84, 1999.

[5] Gagge, A.P., Fobelets, A.P., Berglund, P.E. A standard predictive index of human response to the thermal environment. ASHRAE Trans., 92, 709$731,1986$.

[6] Matzarakis, A. Assessing climate for tourism purposes: Existing methods and tools for the thermal complex. Proceedings of the first international workshop on climate, tourism and recreation, ed. by A. Matzarakis and C. R. de Freitas. International Society of Biometeorology, Commission on Climate Tourism and Recreation. 101-112, 2001.

[7] Matzarakis, A. Climate and bioclimate information for tourism in Greece. Proceedings of the first international workshop on climate, tourism and recreation, ed. by A. Matzarakis and C., 2001.

[8] FANGER, P.; TOFTUM, J. Extension of the PMV model to non-airconditioned buildings in warm climates. Energy and Buildings, n. 34, 533536, 2002.

[9] Tirabassi T., Nassetti S., Short Communication: The representative day, Atmos. Environ., 33, 2427, 1999.

[10] ISO 7730 Moderate thermal environments - determination of the PMV and PPD indices and specification of the conditions for thermal comfort, International Standards Organization, Geneva, 1994. 
846 The Sustainable City VII, Vol. 2

[11] Latini G., Cocci Grifoni R., Tascini S., The Representative Day Technique in the Analysis of Thermal Comfort in Outdoor Urban Spaces, 27th International conference on Passive and Low Energy Architecture, Louvain-la-Neuve, Belgium, 2011.

[12] Bruse, M., The influences of local environmental design on microclimate, PhD Thesis University of Bochum, Bochum, 1999. 